\title{
Size segregation in a granular bore
}

\author{
A.N. Edwards and N.M. Vriend \\ Department of Applied Mathematics and Theoretical Physics, Centre for Mathematical Sciences, \\ The University of Cambridge, Cambridge, CB3 OWA, United Kingdom
}

(Dated: September 12, 2016)

\begin{abstract}
We investigate the effect of particle size segregation in an upslope propagating granular bore. A bidisperse mixture of particles, initially normally graded, flows down an inclined chute and impacts with a closed end. This impact causes the formation of a shock in flow thickness, known as a granular bore, to travel upslope leaving behind a thick deposit. This deposit imprints the local segregated state featuring both pure and mixed regions of particles as a function of downstream position. The particle size distribution through the depth is characterized by a thin purely small particle layer at the base, a significant linear transition region and a thick constant mixed particle layer below the surface, in contrast to previously observed 'S'-shaped steady-state concentration profiles. The experimental observations agree with recent progress that upwards and downwards segregation of large and small particles respectively is asymmetric. We incorporate the three-layer, experimentally observed, size distribution profile into a depth-averaged segregation model to modify it accordingly. Numerical solutions of the new model are able to match our experimental results and therefore motivate the use of a more general particle size distribution profile.
\end{abstract}

\section{INTRODUCTION}

Particle size segregation occurs in granular avalanches by the two processes of 'kinetic sieving' and 'squeeze expulsion' $[1,2]$, whereby small particles preferentially percolate downwards under gravity into gaps that form as the avalanche shears, gradually pushing large particles up to balance the pressure and the solids volume fraction. As a result, large particles tend to rise to the surface of the flow whilst small particles percolate down to the bed, creating an 'inversely graded' size distribution [3].

We study a system of bidisperse granular matter with a 'normally graded' (small particles on top of large) segregated inflow. Particles flow continuously down a smooth inclined chute until they reach the closed end and impact with the wall, which leads to the formation of an upslope propagating granular bore [4, 5] that travels with constant speed until coming to rest when all of the material has been released. Only a handful of controlled experiments that study segregation due to gravity exist [e.g. 6-10]. Our configuration is novel because it ensures that the local segregated state of the avalanche, as a function of distance from $x=0$, is "frozen" in the deposit.

We will analyze the particle size distribution experimentally and derive approximate profiles for the small particle concentration and downslope velocity as functions of height. In this manner we aim to validate recent developments on the asymmetry of particle size segregation, for which several forms asymmetric flux functions have been proposed. The models presented in $[11,12]$ follow from their choice of stress partitioning (the splitting up of the average, or bulk, stress into various contributions, e.g. those of various grainsizes), and a modification thereof based on the reported simulation data, respectively. The authors in [13] explained that many possible flux functions give asymmetric segregation velocities and chose one of the simplest of these, which takes a cubic form. In contrast, [10] observed asymmetric segregation velocities experimentally and adopted this in their model. One of our aims here is to confirm experimentally the asymmetry of the segregation rather than the underlying dominant process that causes it. Future work using various size ratios is required to determine the dominant segregation mechanism.

The experimental particle size distribution and velocity profiles will be assumed invariant and incorporated in a depth-averaged granular flow model [4] with segregation [5]. Previously, 'S'-shaped concentration profiles were determined by [14] as solutions to their segregation-remixing equation for a steady uniform flow, and were also investigated experimentally by [8]. However the form of these solutions is too complicated to incorporate into the depth-averaged model. Previous works [e.g. 5, 9, 15] tend to simply assume an instantaneously, sharply inversely graded concentration profile in order to close the model. Whilst this allows considerable progress to be made once grains have become inversely graded, it is too crude an approximation for flows that do not reach that state quickly. We therefore present an alternative closure by allowing for a range of invariant particle size concentration distributions that are closer to reality, as confirmed by our experimental observations. Lastly, we will solve the new system of equations numerically and compare the results with our experiments. 


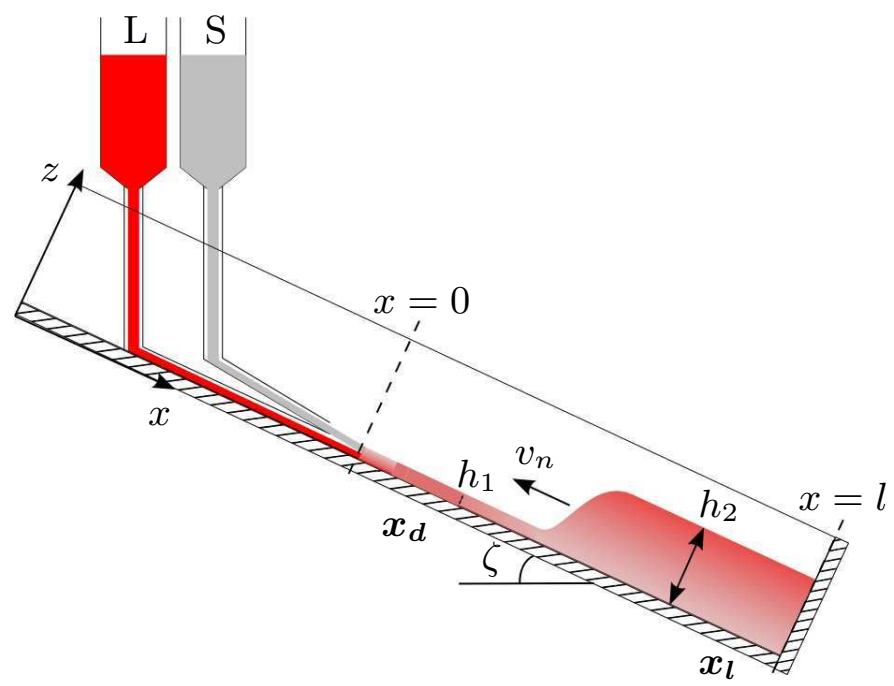

FIG. 1: A sketch of the experimental setup, consisting of a closed, smooth chute of length $126.5 \mathrm{~cm}$ in the downslope $x$-direction from the inflow 'gate' at $x=0$, width $1.5 \mathrm{~cm}$ in the cross-slope $y$-direction (into the page, not shown) and height $4.5 \mathrm{~cm}$ in the normal $z$-direction. The chute is fed by a two-hopper mechanism that allows for the controlled release of 'normally graded' large (L) and small (S) particles. The inflow layer has a thickness $h_{1}$ upstream of the shock, propagating upslope with speed $v_{n}$, which leaves a deposit of thickness $h_{2}$.

\section{EXPERIMENTAL OBSERVATIONS}

Experiments are performed on a closed chute made of smooth perspex, inclined at an angle $\zeta$ to the horizontal. The chute has a length $l=126.5 \mathrm{~cm}$ in the downslope $x$-direction, a width of $1.5 \mathrm{~cm}$ in the cross-slope direction and a height of $4.5 \mathrm{~cm}$ in the normal $z$-direction. A novel two-hopper mechanism is used to fill the chute with material at the upslope end. Opening the hopper gate releases particles from both hoppers simultaneously such that 'small' $0.5-0.6 \mathrm{~mm}$ diameter, silver ballotini from hopper $\mathrm{S}$ fall on top of a layer of 'large' $1-1.18 \mathrm{~mm}$ diameter, red ballotini from hopper L and merge at the inflow 'gate' (an artificial reference point defining $x=0$ ) to form a continuous bidisperse flow that is, in its initial stages, segregated with a normal grading, as shown in fig. 1 . The hoppers are manufactured so that the set flow rate is the same from both hoppers, $Q_{A}=Q_{B}=17.5 \mathrm{gs}^{-1}$, giving a total flow rate of $Q=35 \mathrm{gs}^{-1}$. The particles flow continuously downslope on the smooth bed until reaching the closed end at $x=l$, where impact leads to the formation of an upslope propagating granular bore, defined as a normal (perpendicular to the flow) shock in the flow thickness [4]. The bore propagates with constant speed $v_{n}$, bringing particles to rest on its forwards (downslope) side, until all of the material is released from the hoppers and the flow is stationary everywhere. The height of the bore does not fill the chute in the vertical $z$-direction. The slope inclination angle $\zeta=24.3^{\circ}$ is chosen to coincide with the value found for the angle of repose of both large and small particles (or the mixture thereof), so that the bore leaves a deposit which is parallel to the $x$-axis.

An experiment is carried out and repeated so that video images are captured by a high-speed camera with a rate of 1000fps at two downslope positions; $x_{d}$, at the front of the deposit, around $x=28.2 \mathrm{~cm}$, and $x_{l}$, at the closed end, around $x=126.5 \mathrm{~cm}$. Examples of these images, at two instants for both downslope positions, are shown in fig. 2. The inflow layer is observed to have a thickness $h_{1} \approx 0.5 \mathrm{~cm}$ at $x_{d}$ that thins to $h_{1} \approx 0.4 \mathrm{~cm}$ at $x_{l}$ as the flow accelerates, whilst the bore has a near constant thickness of $h_{2} \approx 1.7 \mathrm{~cm}$. The shock speed is related to $h_{1,2}$ by the hydrodynamic jump condition $[4,16]$,

$$
v_{n}=-\sqrt{g h_{1}\left(h_{1}+h_{2}\right) \cos \zeta / 2 h_{2}}
$$

which has a value of $v_{n}=-0.17 \mathrm{~ms}^{-1}$ at $x_{d}$ and $v_{n}=-0.15 \mathrm{~ms}^{-1}$ at $x_{l}$, i.e. the shock speed decreases with $h_{1}$ for constant $h_{2}$. These agree with the measured experimental values within $\pm 5 \%$. The downslope velocity component $u(x, z, t)$ is measured in the inflow layer by particle image velocimetry (PIV) analysis of the high-speed still images taken during the experiment at the two downslope positions. For modelling of the flow it is assumed that $u$ varies linearly with depth [e.g. 17], i.e.

$$
u=\alpha \bar{u}+2(1-\alpha) \bar{u} z / h
$$

for a parameter $0 \leq \alpha \leq 1$ that allows the profile to vary from simple shear for $\alpha=0$ to plug flow for $\alpha=1$ and linear shear with basal slip for values in-between. The shear parameter is found by fitting the assumed velocity profile (2) 

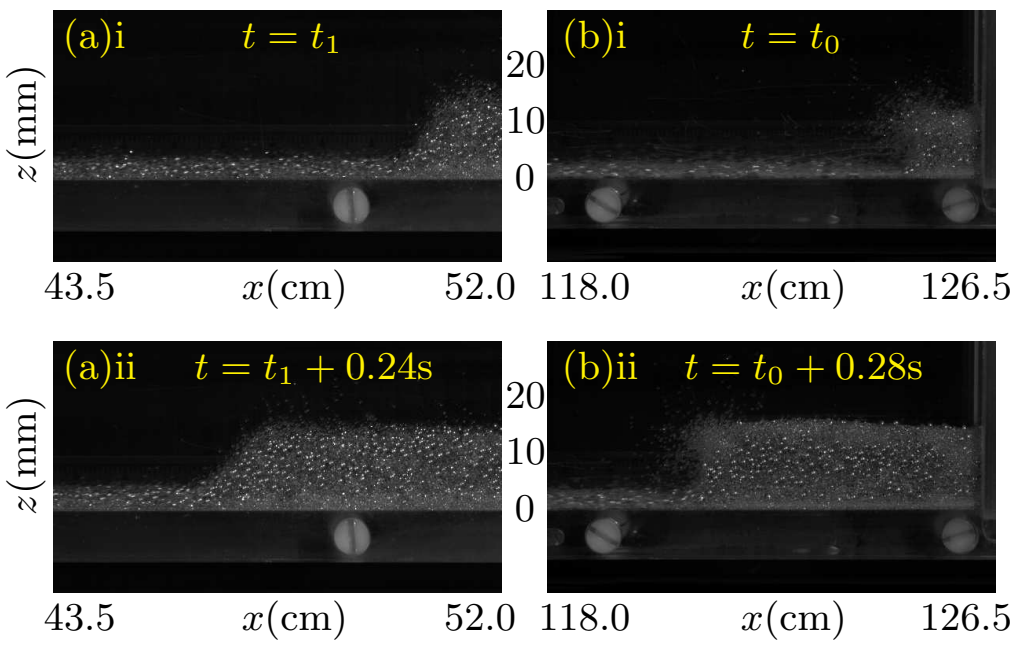

FIG. 2: Still images taken during the experiment at two downslope positions (a) $x_{d}$ and (b) $x_{l}$ for arbitrary times $t_{1}>t_{0}$. The bore is seen to have a smooth, diffusive shock in flow thickness. The segregation process occurs as the bore propagates upslope between positions $x_{d}$ and $x_{l}$.
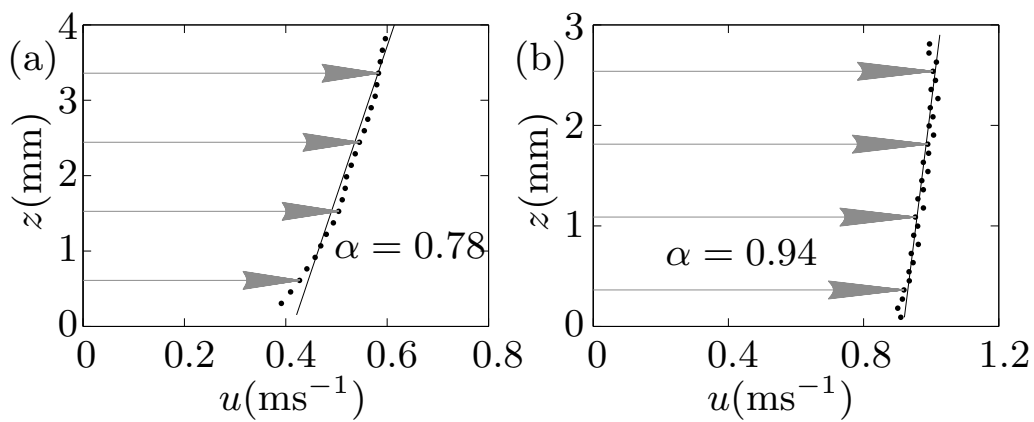

FIG. 3: Measured downslope velocity component $u$ in the inflow layer (dots and illustrative arrows) using PIV analysis at the two downslope positions (a) $x_{d}$ and (b) $x_{l}$. The measurements are averaged over time with a typical standard deviation of $5 \%$ (no greater than $8 \%$ ) from the mean. Data is fitted (solid lines) to the assumed velocity profile (2) by determining the shear rate $\alpha$, whose value is shown.

to the PIV measurements with $\alpha=0.78, \bar{u}=0.54 \mathrm{~ms}^{-1}$ at $x_{d}$ and $\alpha=0.94, \bar{u}=0.97 \mathrm{~ms}^{-1}$ at $x_{l}$, as shown in fig. 3 . As the inflow accelerates, the shear rate increases with $x$ almost to a plug flow and the fast particles spray on impact with the closed end and the bore at $x_{l}$ (fig. $3 \mathrm{~b}$ ), making the shock appear steeper.

After the experiment, an image of the static deposit, shown in fig. 4, is captured through the glass sidewall by traversing a camera parallel to the $x$-axis. The small particle concentration $\phi$ is then measured by analysing the colours in the image to count particles. This is normalized to ensure that there is an equal volume of small and large particles, i.e. the mean concentration in the whole deposit is 0.5 , assuming that the chute is sufficiently thin that cross-channel variation is negligible. The small particle concentration is seen to vary linearly between a purely small particle phase, $\phi=1$, of thickness $\eta$ above the base and a layer with constant $\phi=\phi_{0}$ of thickness $h-\psi$ below the free surface. That is, the concentration profile may be written

$$
\phi= \begin{cases}\phi_{0}, & \psi \leq z \leq h, \\ \left(1-\phi_{0}\right) \frac{\psi-z}{\psi-\eta}+\phi_{0}, & \eta \leq z \leq \psi \\ 1, & 0 \leq z \leq \eta\end{cases}
$$

The depth-averaged small particle concentration $\bar{\phi}$ (as calculated in the following section) and $\phi_{0}$ are both increasing upslope, as the segregation process causes large particles to rise to the surface of the inflow layer, where the downslope velocities and resulting propagation distances are greater. Meanwhile, the constant concentration layer thickness $h-\psi$ is decreasing upslope and the transition region thickness is increasing, as a result of the segregation process occurring over a shorter time and distance. This suggests that the constant concentration layer contains more small particles the further the bore propagates upslope, as time elapses. However, the thickness of the small particle layer $\eta$ remains 


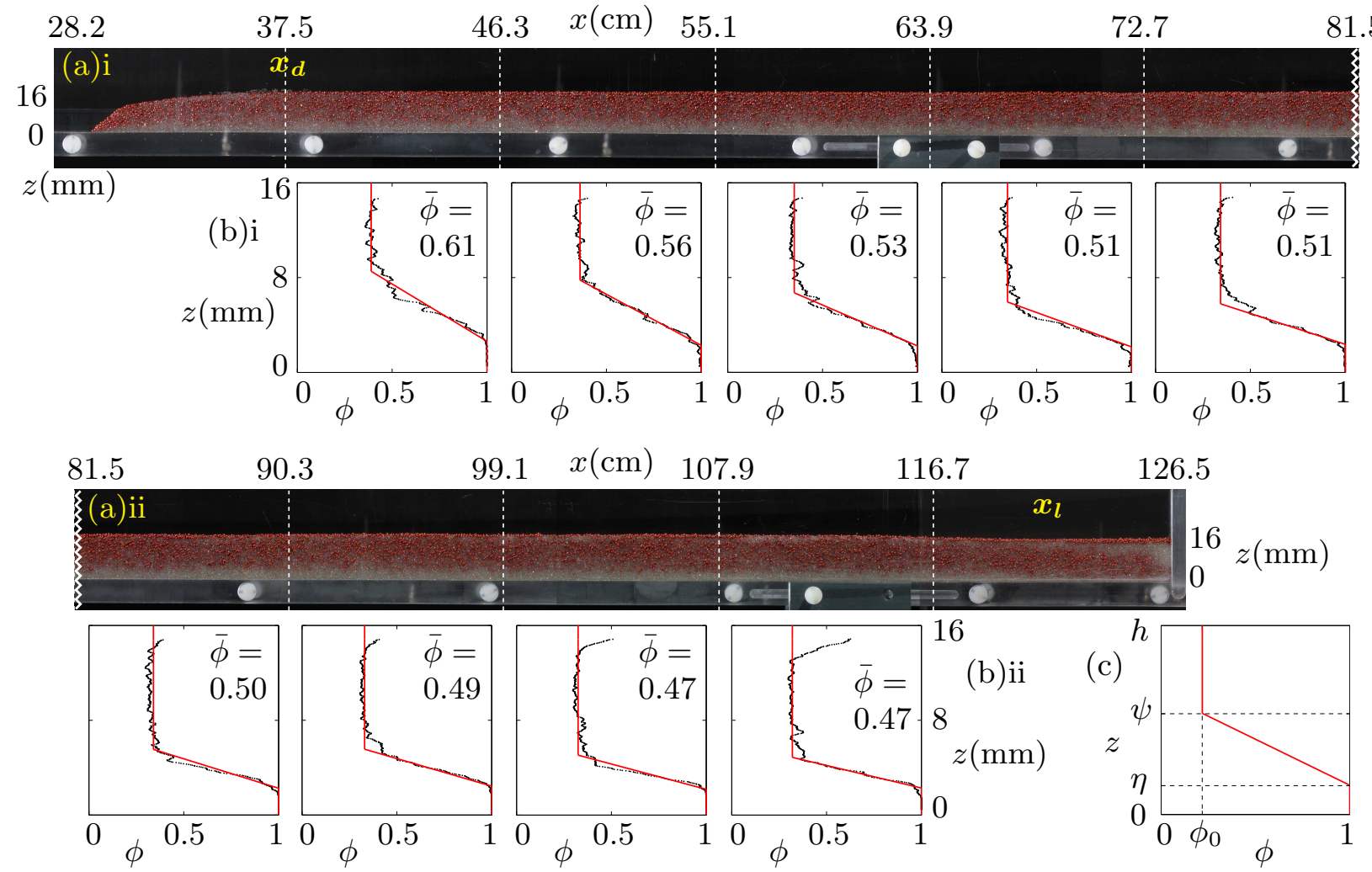

FIG. 4: (a) A still image of the deposit at the end of an experiment, divided into sections (dashed lines) over each of which (b) the measured average small particle concentration $\phi$ profiles is plotted as a function of $z$ (markers) and fitted (solid lines) to the (c) general profile given in equation (3). The image is split into i) $28.2 \mathrm{~cm} \leq x \leq 81.5 \mathrm{~cm}$ and ii) $81.5 \mathrm{~cm} \leq x \leq 126.5 \mathrm{~cm}$ (zigzagged lines) and the downslope positions $x_{d}$ and $x_{l}$ are shown. A full-length horizontal version of this figure is available in the online supplementary material.

roughly constant. Together these observations imply that the flux of small particles downwards occurs faster than the flux of large particles upwards and therefore that the size segregation process is asymmetric, as found by [10-13].

The concentration distribution (3) is more complex than the existing theory [5] that makes the simplest possible assumption that the particle size distribution is sharply inversely graded, i.e. there is a sharp interface between a bottom layer of purely small particles and a top layer of purely large particles, which was based on observations of stratification experiments [6,7]. The form used here instead is a good fit with the experimentally observed concentration profile, whilst still allowing progress to be made in a depth-averaged segregation model. Our profile (3) resembles the 'S'-shaped small particle found experimentally by [8] for a continuous, rough-bedded chute flow with an initial normal grading of particle sizes, which they compared to the theoretical steady-state concentration determined by [14]. Note, however, that such exact theoretical solutions have only been shown to exist [e.g. 14, their eqn. 3.4] for symmetric flux models with diffusion. As such, [13], for example, consider the non-diffuse limit for an analytical solution of their asymmetric flux function.

Furthermore, our experimental findings are supported by those of [10], who also observe (in their fig. 5, around $\hat{t}=30$ ) a layer of small particles, a transition region and a constant concentration layer, which develops during the segregation process.

\section{DEPTH-AVERAGED SEGREGATION MODEL}

Variations in the cross-slope $y$-direction are assumed negligible due to the thinness of the chute and the free-surface of the flow at $z=h(x, t)$ is taken to be a measure of the flow thickness normal to the $x$-axis. The downslope velocity 
$u$ is integrated through the flow thickness as

$$
\bar{u}(x, t)=\frac{1}{h} \int_{0}^{h} u(x, z, t) d z
$$

and the shallowness of the flow is exploited to give the one-dimensional depth-averaged mass and momentum balance equations [e.g. 4]

$$
\begin{gathered}
\frac{\partial h}{\partial t}+\frac{\partial}{\partial x}(h \bar{u})=0 \\
\frac{\partial}{\partial t}(h \bar{u})+\frac{\partial}{\partial x}\left(h \bar{u}^{2}\right)+\frac{\partial}{\partial x}\left(\frac{1}{2} h^{2} g \cos \zeta\right)=S
\end{gathered}
$$

where the source terms

$$
S=h g \cos \zeta(\tan \zeta-\mu)
$$

are comprised of a balance between gravitational acceleration $g$ and a coefficient of basal friction $\mu$. A Savage-Hutter theory [18] is adopted, in which the friction coefficient is simply $\mu=\tan \delta$, for a basal angle of friction $\delta$. It is assumed that basal friction angle is equal to the slope inclination angle, $\delta=\zeta$, which allows for a one-dimensional solution consisting of a travelling discontinuity in flow thickness with constant uniform states on either side [4], as observed experimentally.

The depth-averaged segregation equation may be derived as [see 5, their equation 2.14]

$$
\frac{\partial}{\partial t}(h \bar{\phi})+\frac{\partial}{\partial x}(h \overline{\phi u})=0
$$

where $0 \leq \phi \leq 1$ is the volume fraction of small particles per unit volume and $\bar{\phi}, \bar{\phi} u$ are depth-averaged quantities.

The model is closed by incorporating the experimentally observed downslope velocity profile (2) and the small particle concentration profile (3). The depth-averaged small particle concentration may then be integrated to find

$$
h \bar{\phi}=\int_{0}^{h} \phi \mathrm{dz}=\frac{1-\phi_{0}}{2}(\psi+\eta)+\phi_{0} h .
$$

The depth-averaged flux of small particles can then be calculated as

$$
h \overline{\phi u}=\int_{0}^{h} \phi u \mathrm{dz}=\left(1-\phi_{0}\right)\left[\alpha \bar{u} \eta+(1-\alpha) \bar{u} \frac{\eta^{2}}{h}+\frac{\alpha \bar{u}}{2}(\psi-\eta)+\frac{(1-\alpha) \bar{u}}{3 h}(\psi-\eta)(\psi+2 \eta)\right]
$$

Substituting (9) and (10) into the segregation equation (8), rearranging and using the mass balance equation (5), transforms it into equations for the evolution of the small particle and constant concentration layer thicknesses,

$$
\begin{gathered}
\frac{\partial \eta}{\partial t}+\frac{\partial(\eta \bar{u})}{\partial x}-\frac{\partial}{\partial x}\left((1-\alpha) \bar{u} \eta\left(1-\frac{2 \eta}{3 h}-\frac{\psi}{3 h}\right)\right)=0 \\
\frac{\partial \psi}{\partial t}+\frac{\partial(\psi \bar{u})}{\partial x}-\frac{\partial}{\partial x}\left((1-\alpha) \bar{u} \psi\left(1-\frac{2 \psi}{3 h}-\frac{\eta}{3 h}\right)\right)=0 .
\end{gathered}
$$

The equations have been separated by assuming that the interaction between $\eta$ and $\psi$ is apparent only through the sole cross-product term, whose magnitude is shared equally between them. Each of these are similar to the evolution equation for the inversely graded interface depth in an instantaneously, sharply segregated bidisperse flow [5] that is recovered when $\psi=\eta$ in (11) or (12).

\section{NUMERICAL SIMULATIONS}

The standard depth-averaged avalanche equations [e.g. 4] represent a system of hyperbolic equations that require high-resolution shock capturing numerical methods [e.g. 19] to solve them. This work uses the closely related semidiscrete high-resolution non-oscillatory central schemes of [20], together with the third-order Runge-Kutta adaptive step method [21] for their time evolution. 
In order to solve the system, the depth-averaged equations (5)-(8) are written in terms of conserved variables $h(x, t), m(x, t)=h \bar{u}, \eta(x, t)$ and $\psi(x, t)$ as

$$
\frac{\partial \boldsymbol{w}}{\partial t}+\frac{\partial \boldsymbol{f}(\boldsymbol{w})}{\partial x}=0
$$

where $\boldsymbol{w}=(h, m, \eta, \psi)^{\mathrm{T}}$ is the vector of conserved variables. The resulting convection flux function is

$$
\boldsymbol{f}=\left(\begin{array}{c}
m \\
\frac{m^{2}}{h}+\frac{h^{2}}{2} g \cos \zeta \\
\frac{\eta m}{h}-(1-\alpha) \frac{\eta m}{h}\left(1-\frac{2 \eta}{3 h}-\frac{\psi}{h}\right) \\
\frac{\psi m}{h}-(1-\alpha) \frac{\psi m}{h}\left(1-\frac{2 \psi}{3 h}-\frac{\eta}{h}\right)
\end{array}\right)
$$

The computational domain is one dimensional and defined in the region between the inflow 'gate' at $x=0$ and the closed end at $x=l=126.5 \mathrm{~cm}$. That is $0 \mathrm{~cm} \leq x \leq 126.5 \mathrm{~cm}$ and the domain is discretized over 1265 grid points. The initial conditions are of an empty chute,

$$
h(x, 0)=m(x, 0)=\eta(x, 0)=\psi(x, 0)=0 .
$$

There is a supercritical inflow $(F r>1)$, which requires three boundary conditions [e.g. 22], whilst a no-normal-flux condition is applied at the end wall. The prescribed inflow boundary values of $\eta(0, t)$ and $\psi(0, t)$ are the mean observed experimental values. The value of the depth-averaged velocity at the inflow, $\bar{u}_{0}$, is chosen to allow $\bar{u}$ to accelerate to the experimentally measured value of $\bar{u}=0.54 \mathrm{~ms}^{-1}$ at $x_{d}$, where the fit to the velocity profile $\alpha=0.78$, prior to further acceleration, is used here as the assumed constant shear rate to be representative of the whole flow. This gives the set of boundary conditions

$$
h(0, t)=h_{0}=5 \mathrm{~mm}, \quad \eta(0, t)=0.13 h_{0}, \quad \psi(0, t)=0.40 h_{0}, \quad m(0, t)=\bar{u}_{0} h_{0}=0.4 \mathrm{~ms}^{-1} h_{0}, \quad m(L, t)=0 .
$$

An equal volume of large and small particles is enforced by setting $\bar{\phi}=0.5$ in (9) to give

$$
\phi_{0}=(h-\psi-\eta) /(2 h-\psi-\eta),
$$

which has a value of $\phi_{0}=0.32$ here.

The high-speed experimental images are compared with the numerical simulation at $x_{d}$ and $x_{l}$ in fig. 5 . It is shown that, using the same inflow thickness, the numerics are able to produce a thinning, accelerating inflow layer and a bore with a quantitatively comparable flow thickness and particle layer thicknesses to the experiments. The slope of the shock in the numerics is a result of the grid resolution and the diffusivity of the numerical scheme, and as such it differs from the viscosity of the experimental system.

The numerical results along the whole chute are shown in fig. 6. The small particle layer is coloured silver, the constant concentration layer is coloured red and there is a linear grading between the two colours in the region inbetween. As in the experiments, the inflow layer accelerates and thins before it reaches the closed end and the upslope propagating bore is formed, with an increasing thickness that tends to a steady state. There are three distinct regions where the flow thickness and particle size distributions vary; the inflow layer that, up to thinning, has the same form as the inflow boundary conditions, the main bore region on the downstream side of the shock and an end region that contains an imprint of a jump in $\psi$ where the thin flow front meets the bore. The numerical results are in good agreement with the experimental small particle concentration profile at $x_{d}$ (fig. 6 (b)ii), where the values of $\eta, \psi$ and $\phi_{0}$ are within $2 \%, 10 \%$ and $5 \%$, respectively, of the experimental observations.

\section{CONCLUSIONS}

We have performed laboratory experiments in a closed chute to study the particle size segregation process that occurs in an upslope propagating granular bore. Analysis of the deposit along the entire length of the chute reveals that the particle size distribution consists of a linear transition between a small particle layer at the base and a constant concentration layer below the surface. The form of this profile, and the way it develops as the bore propagates upslope, give support to the recent developments on the asymmetry of particle size segregation [10-13, 23]. The increase in the 

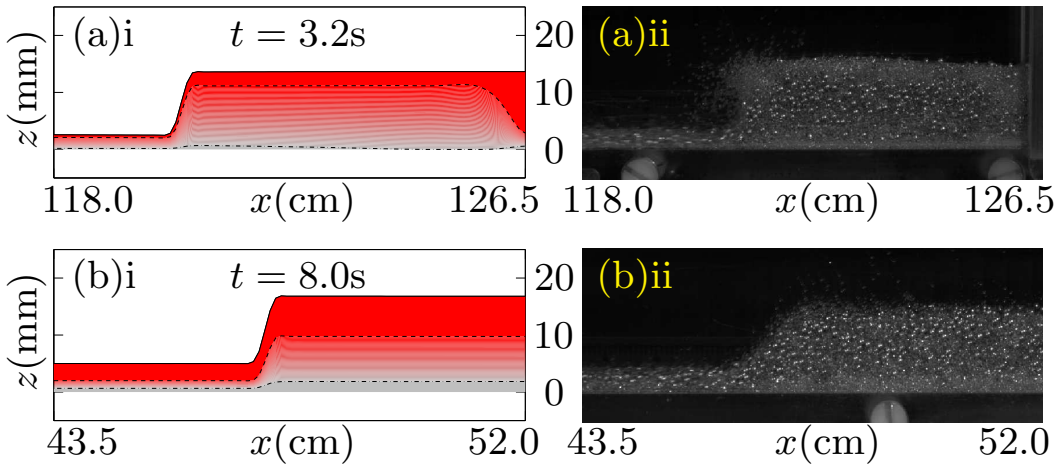

FIG. 5: Comparison between numerics (i) and experiments (ii) at two downslope positions (a) $x_{d}$ and (b) $x_{l}$, both shown with a $1: 1$ aspect ratio of the $x$ - and $z$-axes. In (i), the small particle layer is coloured silver and the constant concentration layer is coloured red, with a linear transition inbetween.

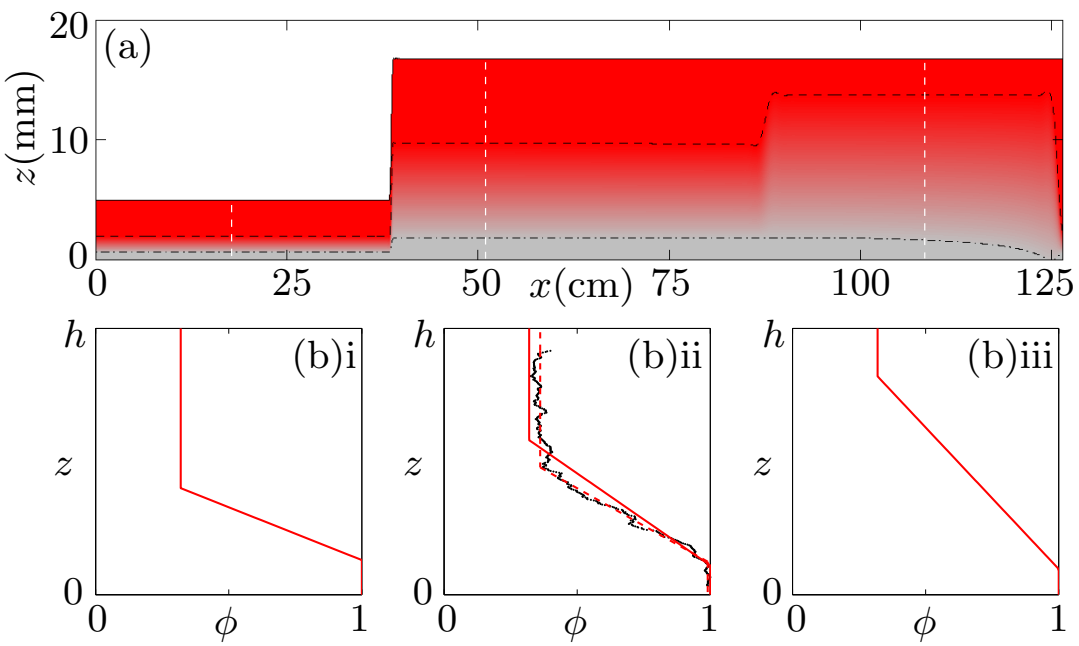

FIG. 6: Results from a numerical simulation for (a) flow thickness $h$ (solid line), small particle $\eta$ (dash-dotted line) and constant concentration $\psi$ (dashed line) layer heights at $t=8.5 \mathrm{~s}$. The small particle layer is coloured silver and the constant concentration layer is coloured red, with a linear transition inbetween. (b) The small particle concentration $\phi$ (solid lines) is plotted for three distinct regions i-iii (dashed white lines). In region ii, numerics are compared with experiments (markers and dashed line) for $46.3 \mathrm{~cm} \leq x \leq 55.1 \mathrm{~cm}$. A movie showing the time-dependent evolution of (a) is available online.

value of the constant concentration $\phi_{0}$ in a layer of decreasing thickness $h-\psi$ as the bore propagates upslope means that there are more small particles in a thinner constant concentration layer as the bore propagation distance increases, since travelling further upslope allows for a shorter time and distance for segregation to occur. The small particle layer height $\eta$ remains approximately constant, however, whilst the depth-averaged small particle concentration $\bar{\phi}$ decreases with increasing downslope position. These observations together imply that the size segregation process is asymmetric, with a greater flux of small particles than large.

Most existing works simply adopt an instantaneously sharply inversely graded profile for the small particle concentration in a depth-averaged segregation model [e.g. 5, 9, 15], which does still allow for considerable progress to be made. 'S'-shaped concentration profiles have also been observed experimentally by $[8]$ that are comparable to steady-state concentrations determined by [14], but these are solutions to a symmetric flux model with diffusion and are also mathematically too complicated to include in the existing depth-averaged segregation model [5]. However, by including our experimentally observed particle size concentration distribution into the depth-averaged segregation model, we have developed a new model which allows for more general particle size distribution profiles. Furthermore, numerical simulations of the new model are able to capture the behaviour of the experiments with good agreement. 


\section{Acknowledgments}

This research was supported by the Returning Carers Scheme of the University of Cambridge. N.M.V. also acknowledges support from her NERC postdoctoral research fellowship NE/I021047/1 and her Royal Society Dorothy Hodgkin Research Fellowship RG130403. The authors would like to thank Stuart Dalziel, Jim McElwaine and Nico Gray for their scientific suggestions.

[1] G. V. Middleton. Experimental studies related to problems of flysch sedimentation. In J. Lajoie, editor, Flysch Sedimentology in North America, pages 253-272, Toronto, 1970. Business and Economics Science Ltd.

[2] S. B. Savage and C. K. K. Lun. Particle size segregation in inclined chute flow of dry cohesionless granular solids. J. Fluid Mech., 189:311-335, 1988 .

[3] G. V. Middleton and M. A. Hampton. Subaqueous sediment transport and deposition by sediment gravity flows. In D. J. Stanley and D. J. P. Swift, editors, Marine sediment transport and environmental management, pages 197-218. Wiley, 1976.

[4] J. M. N. T. Gray, Y. C. Tai, and S. Noelle. Shock waves, dead-zones and particle-free regions in rapid granular free-surface flows. J. Fluid Mech., 491:161-181, 2003.

[5] J. M. N. T. Gray and B. P. Kokelaar. Large particle segregation, transport and accumulation in granular free-surface flows. J. Fluid Mech., 652:105-137, 2010.

[6] J. M. N. T. Gray and K. Hutter. Pattern formation in granular avalanches. Continuum Mech. Es Thermodyn., 9:341-345, 1997.

[7] J. M. N. T. Gray and C. Ancey. Segregation, recirculation and deposition of coarse particles near two-dimensional avalanche fronts. J. Fluid Mech., 629:387-423, 2009.

[8] S. Wiederseiner, N. Andreini, G. Epely-Chauvin, G. Moser, M. Monnereau, J. M. N. T. Gray, and C. Ancey. Experimental investigation into segregating granular flows down chutes. Phys. Fluids, 23:013301, 2011.

[9] M. J. Woodhouse, A. R. Thornton, C. G. Johnson, B. P. Kokelaar, and J. M. N. T. Gray. Segregation-induced fingering instabilities in granular free-surface flows. J. Fluid Mech., 709:543-580, 2012.

[10] K. van der Vaart, P. Gajjar, G. Epely-Chauvin, N. Andreini, J. M. N. T. Gray, and C. Ancey. Underlying asymmetry within particle size segregation. Phys. Rev. Lett., 114:238001, 2015.

[11] B. Marks, P. Rognon, and I. Einav. Grainsize dynamics of polydisperse granular segregation down inclined planes. J. Fluid Mech., 690:499-511, 2012.

[12] D. R. Tunuguntla, O. Bokhove, and A. R. Thornton. A mixture theory for size and density segregation in shallow granular free-surface flows. J. Fluid Mech., 749:99-112, 2014.

[13] P. Gajjar and J. M. N. T. Gray. Asymmetric flux models for particle-size segregation in granular avalanches. J. Fluid Mech., 757:297-329, 2014 .

[14] J. M. N. T. Gray and V. A. Chugunov. Particle-size segregation and diffusive remixing in shallow granular avalanches. J. Fluid Mech., 569:365-398, 2006.

[15] J. M. N. T. Gray, P. Gajjar, and B. P. Kokelaar. Particle-size segregation in dense granular avalanches. C. R. Physique, $16: 73-85,2015$.

[16] J. J. Stoker. Water waves. Interscience, 1957.

[17] J. M. N. T. Gray and A. R. Thornton. A theory for particle size segregation in shallow granular free-surface flows. Proc. Roy. Soc. A, 461:1447-1473, 2005.

[18] S. B. Savage and K. Hutter. The motion of a finite mass of granular material down a rough incline. J. Fluid Mech., 199:177-215, 1989

[19] H. Nessyahu and E. Tadmor. Non-oscillatory central differencing for hyperbolic conservation laws. J. Comput. Phys., 87:408-463, 1990.

[20] A Kurganov and E. Tadmor. New high-resolution central schemes for nonlinear conservation laws and convection-diffusion equations. J. Comput. Phys., 160:241-282, 2000.

[21] A. A. Medovikov. High order explicit methods for parabolic equations. BIT, 38(2):372-390, 1998.

[22] T. Weiyan. Shallow water hydrodynamics. Elsevier, New York, 1992.

[23] L. A. Golick and K. E. Daniels. Mixing and segregation rates in sheared granular materials. Phys. Rev. E, 80(4):042301, Oct 2009 . 